\title{
The methods and use of questionnaires
} for the diagnosis of dental phobia by Japanese dental practitioners specializing in special needs dentistry and dental anesthesiology: a cross-sectional study

\author{
Mika Ogawa' ${ }^{1}$,Terumi Ayuse ${ }^{2^{*}}$, Toshiaki Fujisawa ${ }^{3}$, Shuntaro Sato ${ }^{4}$ and Takao Ayuse ${ }^{5}$
}

\begin{abstract}
Background: Dental phobia is covered by medical insurance; however, the diagnostic methods are not standardized in Japan. Therefore, the aim of this study was to investigate the methods and use of questionnaires for the diagnosis of dental phobia by Japanese dental practitioners specializing in special needs dentistry and dental anesthesiology.

Methods: We conducted an online survey to obtain information from the members of the Japanese Society for Disability and Oral Health (JSDH, $n=5134$ ) and the Japanese Dental Society of Anesthesiology (JDSA, $n=2759$ ). Response items included gender, qualification, affiliation type, methods of diagnosis and management of dental phobia, use of questionnaire, need to establish standardized diagnostic method for dental phobia, and others. The chi-squared test was used to compare answers between the three groups: JSDH only, JDSA only, and both JSDH and JDSA. Multiple logistic regression analysis was conducted to identify factors associated with the use of an assessment questionnaire.

Results: Data were obtained from 614 practitioners (JSDH only, $n=329$; JDSA only, $n=195$; both JSDH and JDSA: $n=90$, response rate: $7.8 \%[614 / 7,893]$, men: $n=364$ [58.5\%]). Only 9.7\% of practitioners used questionnaires to quantify the level of dental anxiety. The members of both JSDH and JDSA group used questionnaires more frequently than members of the JSDH only ( $19 \%$ and $7.1 \%$, respectively; Bonferroni corrected $p<0.01)$. Most practitioners $(89.1 \%)$ diagnosed dental phobia based on patient complaints of fear of treatment. Furthermore, majority of the participants (73.3\%) felt the need to establish standardized diagnostic method for "dental phobia." Multiple logistic regression analysis showed that membership of the JSDH only was negatively related (odds ratio [OR] 0.28, 95\% confidence interval [CI] 0.13-0.60), and use of behavioral therapy was positively related (OR 2.34, 95\% $\mathrm{Cl} 1.18-4.84)$ to the use of a questionnaire.

Conclusions: The results of this study showed that the use of questionnaires was very low, patients' subjective opinions were commonly used to diagnose dental phobia, and a standardized diagnostic criterion was thus needed among practitioners. Therefore, it is necessary to establish diagnostic criteria for dental phobia in line with the Japanese clinical system and to educate dentists about them.
\end{abstract}

\footnotetext{
*Correspondence: ttagawa@nagasaki-u.ac.jp

2 Department of Special Care Dentistry, Nagasaki University Hospital,

Nagasaki, Japan

Full list of author information is available at the end of the article
} original author(s) and the source, provide a link to the Creative Commons licence, and indicate if changes were made. The images or other third party material in this article are included in the article's Creative Commons licence, unless indicated otherwise in a credit line to the material. If material is not included in the article's Creative Commons licence and your intended use is not permitted by statutory regulation or exceeds the permitted use, you will need to obtain permission directly from the copyright holder. To view a copy of this licence, visit http://creativecommons.org/licenses/by/4.0/. The Creative Commons Public Domain Dedication waiver (http://creativeco mmons.org/publicdomain/zero/1.0/) applies to the data made available in this article, unless otherwise stated in a credit line to the data. 
Keywords: Dental phobia, Questionnaire, Special needs dentistry, Dental anesthesiology, Dental practitioner

\section{Background}

Dental phobia is recognized as extremely high anxiety for dental treatment. In such cases, it is difficult to perform dental treatments using routine methods [1, 2]. Therefore, dental treatments for patients with dental phobia can be performed by utilizing a pharmacological approach, such as cognitive behavioral therapy (CBT) or a psychological approach such as sedation [2, 3]. "Dental phobia" is covered by health insurance in Japan; hence, the term is highly recognized by general dentists. Dental phobia can be accordingly diagnosed in two ways.

The first is a diagnosis according to the Diagnostic and Statistical Manual of Mental Disorders, Fifth Edition (DSM-5). Dental phobia is a specific type of phobia (localized phobia) in the DSM-5 [3]. Specific phobia is a condition in which a person feels fear or anxiety about a specific situation or subject. Diagnosis is based on interviews or self-administered questionnaires formulated according to the DSM-5 criteria. The incidence of dental phobia based on DSM classification is $2.1 \%$ (Sweden) [4], 3.1\% (USA) [5], and 3.7\% (Netherlands) [6]. A diagnosis of dental phobia according to the DSM-5 criteria is often made by doctors or psychologists; however this is not common in Japan.

The second method is to diagnose dental phobia as severe dental anxiety or fear, and to use the cut-off values of the questionnaire. The concept of dental anxiety or fear is a continuum rather than a dichotomy of scary/not scary. Quantification is essential for determining methods of dealing with dental anxiety or fear $[7,8]$. Therefore, multiple psychological scales with established reliability and validity have been developed and used worldwide [1-3]. For example, the Modified Dental Anxiety Scale (MDAS) is a five-item questionnaire that quantifies the degree of anxiety in five dental situations [9]. The total score ranges from 5 to 25 . An MDAS score of $\geq 19$ indicates a high level of dental anxiety and specific phobia [10]. The prevalence of high dental fear (MDAS score $\geq 19$ ) is approximately $10 \%$ in Japan [11] and in other countries [9, 12, 13]. However, the concept of dental anxiety is less recognized by Japanese dentists than dental phobia, and the measurement of dental anxiety using questionnaires is not common in Japan.

The treatment of dental phobia varies across countries. In Scandinavian countries, dental phobia is mainly diagnosed by psychologists, and CBT by psychologists is conducted in specialized facilities [14,
15]. Sedation in primary dental care is common in the UK [16]. However, the importance of CBT for dental phobia has also been pointed out, and it has been reported that psychologist-led CBT services are being provided accordingly $[17,18]$. In Japan, however, CBT by psychologists is rarely used. Some general dental clinics offer systematic desensitization methods, such as tell-show-do and nitrous oxide inhalation sedation by general dentists. Dental phobia patients who cannot be treated by general dentists are referred to higher medical institutions, such as university hospitals and regional clinics. These institutions often have departments of special-needs dentistry and dental anesthesiology. Experienced dentists are often certified dentists or specialists of the Japanese Society for Disability and Oral Health (JSDH) and the Japanese Dental Society of Anesthesiology (JDSA).

Typical criteria for JSDH specialists include completion of a training program of at least 5 years in total at a prescribed training facility. Opportunities to practice dentistry for the disabled should be routine. JDSA's criteria for specialists include being a member of the JDSA for $>5$ years, being in the field of dental anesthesia, and completing the specialist training curriculum. Therefore, the difference in patient demographics between the two organizations is that JSDH specialists typically treat patients with disabilities, while JDSA specialists typically provide general anesthesia, sedation, and pain clinic services for dental treatment and oral surgery. Patients with dental phobia who are referred to higher medical institutions are often treated by JSDH members under sedation or general anesthesia performed by JDSA members $[19,20]$. Therefore, it is likely that JSDH and JDSA members have more contact with dental phobia patients than other dentists. However, the measurement of dental anxiety is not included in the training curriculum of the JSDH and JDSA.

The term dental phobia is commonly used among general dentists and even among patients in Japan; however, a clear definition or diagnostic criterion for dental phobia has not been established till date. Therefore, the diagnosis of dental phobia is considered to depend on the experience of dentists in Japan. The aim of this study was to investigate the methods and use of questionnaires for the diagnosis of "dental phobia" by Japanese dental practitioners specializing in special needs dentistry and dental anesthesiology. We hypothesized that the diagnosis of dental phobia in our participants would be subjective, that the use of 
questionnaires would be low, and that they would want to establish standardized diagnostic criteria.

\section{Methods}

Members of the JSDH (total number of members: 5,134) and the JDSA (total number of members: 2759) were targeted for this cross-sectional study. All the methods were performed in accordance with the Declaration of Helsinki. All experimental protocols, including the method of obtaining consent from the participants, were approved by the respective boards of both academic societies (No. 1920-7 from the JDSA and No. 20029 from JSDH). We conducted an online questionnaire survey (total number of requests: 7893) using Google Forms (California, Google LLC). The web address for the questionnaire survey was sent to all members via e-mail newsletters of both academic societies. Participation in the survey was voluntary and anonymous. The aim and other details of the study were explained on the first screen. Clicking on the button located at the bottom of the first screen by the participant was taken as informed consent. The reminder was given twice via e-mail newsletters to the participants. Anonymous responses between August 7, 2020, and August 22, 2020, were recorded on the Internet.

The questionnaire included the following items:

(1) Gender (male, female)

(2) Qualification (JSDH: specialist [required years of experiences $=5$ years]; certified doctor $[3$ years]; certified dental hygienist [3 years]; other, JDSA: specialist [5 years]; certified doctor [ 2 years]; certified dental hygienist [ 1 year], others [non-certified dentists and hygienist])

(3) Affiliation type (individual clinic, university hospital, regional clinic, hospital dentistry)

(4) Method for management (behavioral therapy, intravenous sedation, general anesthesia, nitrous oxide inhalation sedation, oral sedation, etc.)

(5) Actual diagnostic criteria for dental phobia in new patients (patient complaints of anxiety, experience of feeling unwell during treatment, difficulty in keeping the patient's mouth open during treatment, body movement during treatment, cancellation of dental reception, etc.)

(6) Use of a questionnaire or criteria to diagnose dental phobia (yes/no)

(7) Dental anxiety assessment indices used (modified Dental Anxiety Scale, Dental Fear Survey, Short version of the Dental Anxiety Inventory, Dental Subscale of Children's Fear Survey Schedule, Visual Analog Scale, State-Trait Anxiety Inventory, StateTrait Anxiety Inventory for Children, and original evaluation criteria)
(8) The need to establish standardized diagnostic method for dental phobia (Do you think it is necessary to establish standardized assessment criteria for dental phobia?-very needed, a little needed, either, not needed a little, no need at all) and

(9) Percentage of patients with dental phobia treated every week.

\section{Eligibility criteria}

The inclusion criterion was participants who did not respond to their qualification. There are no exclusion criteria.

\section{Classification of groups}

The groupings are defined as follows: members of the "JSDH only" group belong to JSDH but not to JDSA, those of "JDSA only" group belong to JSDA but not to JDSH, and those of "both JSDH and JDSA" group belong to both JSDH and JDSA.

\section{Statistical analysis}

The characteristics and questionnaire answers were summarized as counts and percentages. Fisher's exact test was used to examine the association between participants' qualifications and whether they used a questionnaire. The chi-squared test or Fisher's exact test was used to compare answers between the three groups (JSDH only, JDSA only, and both JSDH and JDSA). The two groups were then compared using the same test, and the $\mathrm{P}$ value was corrected for Bonferroni correction. Multiple logistic regression analysis was conducted to identify factors associated with the use of a dental anxiety assessment questionnaire and estimated odds ratios (ORs), 95\% confidence intervals (95\% CIs), and p-values. This model included gender, membership, facility type, and five types of management as an independent factor. All tests were conducted at a significance level of 0.05 . All statistical analyses were performed with $\mathrm{R}$ version 4.0.0 ( $\mathrm{R}$ Foundation for Statistical Computing, Vienna, Austria).

\section{Results}

A total of $7.88 \%(622 / 7,893)$ of the participants responded to the survey. Eight responses had missing qualification data; therefore, 614 responses were finally included in the study. Fifty-eight participants were male (356/614). Almost 10\% of the participants (66/614) calculated that the proportion of patients with dental phobia treated every working week was $25 \%$ and above. Table 1 shows the relationship between the qualifications of the participants and the use of questionnaires to diagnose dental phobia. Thirteen percent of the JSDH only group members were specialists (53/419), 
Table 1 Qualification of the participants and the usage of questionnaire to diagnose dental phobia

\begin{tabular}{|c|c|c|c|c|c|c|c|c|}
\hline & & JSDH & & & JDSA & & & \\
\hline & & $\begin{array}{l}\text { The use of } \\
\text { questionnaire } \\
\text { n (\%) }\end{array}$ & & & $\begin{array}{l}\text { The use of } \\
\text { questionnaire } \\
n(\%)\end{array}$ & & & \\
\hline Qualification & Overall n (\%) & No & Yes & $P$ & Overall & No & Yes & $\mathrm{P}$ \\
\hline Specialist & $53(12.8)$ & $48(12.8)$ & $5(12.5)$ & 0.97 & $109(38.2)$ & 99 (39.9) & $10(27.0)$ & 0.049 \\
\hline Certified doctors & $193(46.5)$ & $174(46.4)$ & $19(47.5)$ & & $132(46.3)$ & $115(46.4)$ & $17(45.9)$ & \\
\hline Certified dental hygienist & $32(7.7)$ & $30(8.0)$ & $2(5.0)$ & & $7(2.5)$ & $7(2.8)$ & $0(0.0)$ & \\
\hline Others & $137(33.0)$ & $123(32.8)$ & $14(35.0)$ & & $37(13.0)$ & $27(10.9)$ & $10(27.0)$ & \\
\hline Total & $419(100)$ & $375(100)$ & $40(100)$ & & $285(100)$ & $248(100)$ & $37(100)$ & \\
\hline
\end{tabular}

JSDH = Japanese Society for Disability and Oral Health, JDSA = Japanese Dental Society of Anesthesiology

whereas $38.2 \%$ of the JDSA only group members were specialists $(109 / 285)$. There was no statistically significant association between participants' qualifications and the use of a questionnaire among JSDH only group members $(p=0.97)$. However, there was a slightly significant association observed between the JDSA only group members $(\mathrm{p}=0.049)$.

The common answers and differences among members are shown in Table 2. The percentages of affiliated facility type, use of questionnaires, actual diagnostic criteria, need for standardized diagnostic methods, and methods of management were significantly different between the groups. Approximately one-third of the respondents worked at an individual clinic, and one-third worked at a university hospital. Behavioral therapy was significantly used more frequently among members of the JSDH only (JSDH only group, 264/329 [80\%]) than among members of the JDSA only (JDSA only group, 66/195 [34\%], corrected $\mathrm{p}<0.01)$. Intravenous sedation was significantly more common in the JDSA only group than in the JSDH only group (170/195 [87\%] and 162/329 [49\%], respectively; corrected $\mathrm{p}<0.01)$.

Only $9.8 \%(60 / 614)$ of the participants used a questionnaire to diagnose dental phobia. The members of the both JSDH and JDSA group used questionnaires more frequently than members of the JSDH only group (17/90 [19\%] and 23/329 [7.1\%], respectively; corrected $\mathrm{p}<0.01)$. Eighty-nine percent $(546 / 614)$ of participants diagnosed dental phobia based on patient complaints of anxiety, and 65\% (393/614) by experience of feeling unwell during treatment. Difficulty in keeping the patient's mouth open during treatment was used more frequently as a diagnostic criterion among the JSDH only group members than among the JDSA only group members (101/329 [31\%] and 38/195 [19\%], respectively; corrected $\mathrm{p}=0.02$ ). Body movement during treatment was also used more commonly as a diagnostic criterion among the JSDH only group members than among the JDSA only group members $(229 / 329$ [70\%] and 108/195 [55\%], respectively; corrected $\mathrm{p}<0.01$ ).

Table 3 shows the various dental anxiety assessment questionnaires used by practitioners. Sixty-four percent of the respondents (39/60) who used questionnaires used the original evaluation criteria for the diagnosis of dental anxiety.

Seventy-three percent of respondents (446/612) felt that an established standardized diagnostic method for dental phobia was needed accordingly (Table 2). A greater number of members of the JSDH only group felt the need for standardized diagnostic methods than those of the JDSA only group (257/329 [78\%] and 127/195 [65\%], respectively; corrected $\mathrm{p}<0.01)$.

\section{Multiple regression analysis}

Table 4 shows the associations between the use of dental anxiety assessment questionnaires and participants' characteristics. Eleven participants were excluded due to missing data on the variables used, and 603 participants were hence finally included in the analysis. The factors that found a statistically significant association with the use of dental anxiety assessment questionnaires were membership of the JSDH only group (OR $0.28,95 \% \mathrm{CI}$ $0.13-0.60, \mathrm{p}=0.001)$ compared to both JSDH and JDSA and use of behavioral therapy (OR 2.34, 95\% CI 1.18$4.84, \mathrm{p}=0.018)$.

\section{Discussion}

This study investigated the methods of diagnosis of dental phobia using an online survey among Japanese dental practitioners who specialize in special-needs dentistry and dental anesthesiology. The results showed that the use of questionnaires was very low, patients' subjective opinions were mostly used to diagnose dental phobia, and establishment of standardized diagnostic criteria was 
Table 2 The difference on the answer between memberships

\begin{tabular}{|c|c|c|c|c|c|}
\hline & Overall & JSDH only & JDSA only & $\begin{array}{l}\text { Both JSDH and } \\
\text { JDSA }\end{array}$ & $\mathrm{P}^{*}$ \\
\hline & $N=614$ & $N=329$ & $\mathrm{~N}=195$ & $\mathrm{~N}=90$ & \\
\hline & n (\%) & n (\%) & n (\%) & n (\%) & \\
\hline Gender & & & & & 0.074 \\
\hline Male & $356(58)$ & $197(61)$ & $101(52)$ & $58(64)$ & \\
\hline Female & $253(42)$ & $128(39)$ & $93(48)$ & $32(36)$ & \\
\hline (Missing) & 5 & 4 & 1 & 0 & \\
\hline Affiliation type & & & & & 0.041 \\
\hline Individual clinic & $223(36)$ & $122(37)$ & $73(37)$ & $28(31)$ & \\
\hline University hospital & $194(32)$ & $88(27)$ & $71(36)$ & $35(39)$ & \\
\hline Regional clinic & $99(16)$ & $64(19)$ & $20(10)$ & $15(17)$ & \\
\hline Hospital dentistry & $98(16)$ & $55(17)$ & $31(16)$ & $12(13)$ & \\
\hline The use of a questionnaire & & & & & 0.004 \\
\hline Yes & $60(9.8)$ & $23(7.1)$ & $20(10)$ & $17(19)$ & \\
\hline No & $550(90)$ & $302(93)$ & $175(90)$ & $73(81)$ & \\
\hline (Missing) & 4 & 4 & 0 & 0 & \\
\hline \multicolumn{6}{|l|}{ Actual criteria for diagnosing dental phobia in new patients } \\
\hline Patient's complaining of anxiety & $546(89)$ & $287(87)$ & $177(91)$ & $82(91)$ & 0.400 \\
\hline Experience of feeling unwell during treatment & $397(65)$ & $206(63)$ & $131(67)$ & $60(67)$ & 0.500 \\
\hline Difficulty in keeping the patient's mouth open during treatment & $174(28)$ & $101(31)$ & $38(19)$ & $35(39)$ & 0.001 \\
\hline Body movement during treatment & $391(64)$ & $229(70)$ & $108(55)$ & $54(60)$ & 0.003 \\
\hline Cancellation of dental reception & $149(24)$ & $80(24)$ & $46(24)$ & $23(26)$ & $>0.9$ \\
\hline Need for establishment of standardized diagnostic method & & & & & 0.004 \\
\hline Very needed/a little needed & $446(73)$ & $257(78)$ & $127(65)$ & $62(69)$ & \\
\hline Either/not needed a little/no need at all & $168(27)$ & $72(22)$ & $68(35)$ & $28(31)$ & \\
\hline \multicolumn{6}{|l|}{ Method for management } \\
\hline Behavioral therapy & $384(63)$ & $264(80)$ & $66(34)$ & $54(60)$ & $<0.001$ \\
\hline Intravenous sedation & $412(67)$ & $162(49)$ & $170(87)$ & $80(89)$ & $<0.001$ \\
\hline General anesthesia & $283(46)$ & $125(38)$ & $98(50)$ & $60(67)$ & $<0.001$ \\
\hline Nitrous oxide inhalation sedation & $296(48)$ & $165(50)$ & $80(41)$ & $51(57)$ & 0.028 \\
\hline Oral sedation & $55(9.0)$ & $17(5.2)$ & $23(12)$ & $15(17)$ & $<0.001$ \\
\hline Others & $25(4.1)$ & $17(5.2)$ & $6(3.1)$ & $2(2.2)$ & 0.400 \\
\hline (Missing) & 1 & 0 & 1 & 0 & \\
\hline
\end{tabular}

JSDH = Japanese Society for Disability and Oral Health, JDSA = Japanese Dental Society of Anesthesiology

*Pearson's Chi-squared test

Table 3 Questionnaires used by respondents

\begin{tabular}{ll}
\hline Questionnaires & $\mathbf{n}(\%)$ \\
\hline Modified Dental Anxiety Scale & $9(15.1)$ \\
Dental Fear Survey & $6(10.3)$ \\
The short version of the Dental Anxiety Inventory & $2(2.4)$ \\
Dental Subscale of Children's Fear Survey Schedule & $1(1.6)$ \\
Visual Analog Scale & $12(19.8)$ \\
State-Trait Anxiety Inventory & $4(6.3)$ \\
State-Trait Anxiety Inventory for Children & $1(1.6)$ \\
Original evaluation criteria & $39(64.3)$
\end{tabular}

Respondents were able to choose more than one needed among practitioners. These results supported our hypothesis.

Only $9.7 \%$ of the practitioners included in the analysis who specialized in special-needs dentistry and dental anesthesiology used questionnaires to quantify the level of dental anxiety. These findings can be compared with those of the UK- and Australia-based studies. Among practitioners interested in behavioral sciences in the UK, $20 \%$ used questionnaires [21], and 3.4\% of general dentists in Australia used published scales [22]. The difference between the results of our study and those of these studies could be explained by the differences in 
Table 4 Results of a logistic regression analysis for the usage of questionnaire to diagnose dental phobia

\begin{tabular}{llll}
\hline Variables & OR & $\mathbf{9 5 \%} \mathbf{C l}$ & $\mathbf{P}$ \\
\hline $\begin{array}{l}\text { Gender } \\
\quad \text { Female }\end{array}$ & - & - & \\
$\quad$ Male & 1.78 & $0.98,3.32$ & 0.063 \\
Memberships & & & \\
$\quad$ Both JSDH and JDSA & - & - & - \\
$\quad$ JSDH only & 0.28 & $0.13,0.60$ & 0.001 \\
$\quad$ JDSA only & 0.63 & $0.30,1.35$ & 0.23 \\
Specialist & 0.60 & $0.29,1.20$ & 0.16 \\
Affiliated facility type & 1.34 & $0.67,2.74$ & 0.42 \\
Behavioral therapy & 2.34 & $1.18,4.84$ & 0.018 \\
Intravenous sedation & 1.41 & $0.66,3.10$ & 0.38 \\
General anesthesia & 1.24 & $0.64,2.49$ & 0.53 \\
Nitrous oxide inhalation sedation & 0.94 & $0.52,1.71$ & 0.85 \\
Oral sedation & 0.91 & $0.34,2.15$ & 0.84 \\
\hline
\end{tabular}

$\mathrm{OR}=$ Odds Ratio, $\mathrm{Cl}=$ Confidence Interval, JSDH = Japanese Society for Disability and Oral Health, JDSA = Japanese Dental Society of Anesthesiology

the composition of the study population. Nonetheless, the use of questionnaires for the diagnosis of dental anxiety is uncommon among dentists in Japan.

In this study, we observed that almost $90 \%$ of the participants diagnosed dental phobia based on complaints of fear of dental treatment, which is a similar finding to that of a previous study conducted in Australia, which showed that almost one-half of dentists directly asked their patients about dental anxiety [22]. Several standardized methods for the objective and quantitative evaluation of dental phobia are clinically applied; however, dentists continue to diagnose dental phobia or high-level dental anxiety based on subjective and original methods.

The results of multivariate analysis showed that membership of the JSDH only group was negatively related to the use of a questionnaire compared to the both JSDH and JDSA group. In addition, we found that members of the JSDH only group were more likely to diagnose dental phobia by the patient's body movements than other groups, and that they also had a greater need to establish standardized diagnostic criteria. There are two possible reasons why the JSDH only group members did not use the questionnaire. First, as mentioned above, the concept of measuring dental anxiety is not common in Japan. This is because it is not part of the model core curriculum, which is the government's summary of the minimum educational content before graduation [23]. Therefore, dentists may not know that a questionnaire has been developed for this purpose. Another reason for this is the patient population.
Members of the JSDH only group would treat more patients with disabilities combined with dental phobia than those with dental phobia but no disability. Many of the questionnaires that have been developed are selfadministered; therefore, they are difficult to apply to patients with severe disabilities. A study using a validated questionnaire to measure dental fear in patients with mild to moderate intellectual disability reported that patients with a higher degree of intellectual disability had a higher level of dental fear [24]. The results of this study may suggest that the development of new diagnostic methods for dental fear in patients with disabilities is needed among dentists who specialize in special-needs dentistry. This study did not investigate the reasons why the participants did not use the existing questionnaire. Further investigation is needed to determine why they are not used and what diagnostic criteria are needed.

In contrast, these results also revealed that the use of behavioral therapy was positively related to the use of a questionnaire. Behavioral therapy is a psychological approach for the management of dental phobia [2]; therefore, analysis of dental anxiety using published tools would be common among dentists who use behavioral therapy. A previous study reported that female dentists and dentists who used the sedation method tended to use questionnaires more frequently [21]. However, these tendencies were not observed in the present study. Further research using larger samples is required accordingly.

The presence or absence of a specialist qualification (the required years of experience $=5$ years) was not associated with the use of the questionnaire. In Australia, younger dentists are more likely to have received education related to the diagnosis and treatment of dental phobia and to report greater concerns about dental anxiety [22]. Age as a factor was not included in this study; however, it is expected that members who have a specialization are older than those who do not. Furthermore, in Japan, education on dental anxiety seems to be inadequate because the model core curriculum does not include items related to dental anxiety and dental phobia [23]. These factors would suggest that the age of dentists was not related to their interest in dental phobia in Japan.

The main limitation of this study is the low response proportion (7.8\%); therefore, our results may not be generalizable to all members of the JDSA and JSDH. It is possible that the members who participated in the study were more likely to have an interest in dental phobia than those who did not participate. The target population may have used fewer questionnaires and may have had a lesser need for a standardized diagnostic method for dental phobia. The number of subjects using the questionnaire was also small (60 participants). Therefore, the effect of random errors may be present in factors related to the 
use of the questionnaire. To overcome this issue, it is necessary to conduct a large-scale survey. However, this is the first study to assess the methods of diagnosis for dental phobia used by dentists who specialize in specialneeds dentistry and dental anesthesiology.

\section{Conclusions}

In our study population, the use of questionnaires was very low, patients' subjective opinions were commonly used to diagnose dental phobia, and establishment of standardized diagnostic criteria were needed among the practitioners. Therefore, it is necessary to establish diagnostic criteria in line with the Japanese clinical system for dental phobia and to educate dentists about the criteria.

\author{
Abbreviations \\ CBT: Cognitive Behavioral Therapy; DSM-5: Diagnostic and Statistical Manual of \\ Mental Disorders, Fifth Edition; JSDH: Japanese Society for Disability and Oral
} Health; JDSA: Japanese Dental Society of Anesthesiology.

\section{Acknowledgements}

The authors thank Shouji Hironaka, Chairman of the Japanese Society for Disability and Oral Health, and Takehiko lijima, Chairman of the Japanese Dental Society of Anesthesiology, for supporting the dissemination of the survey.

\section{Authors' contributions}

MG conceptualized, investigated, interpreted the data, prepared the original draft, and wrote the manuscript. TA conceptualized, investigated, and prepared the original draft and wrote the manuscript. TF conceptualized, reviewed, and edited the draft. SS interpreted the data and performed the statistical analysis. TA arranged the research grant, conceptualized, investigated, interpreted the data, prepared the original draft, and wrote the manuscript. All authors read and approved the final manuscript.

\section{Funding}

This research (the design of the study and collection, analysis, and interpretation of data and in writing the manuscript) was conducted by the Japanese Dental Society of Anesthesiology with a research grant from the Japanese Association for Dental Science (Issue number: JDSF-DSP1-2020-111-1, task name: Research on the disease concept of dental phobia).

\section{Availability of data and materials}

The datasets used and/or analyzed during the current study are available from the corresponding author upon reasonable request.

\section{Declarations}

\section{Ethics approval and consent to participate}

The research project was approved by the Research Ethics Committees (No. 1920-7 from the Japanese Dental Society of Anesthesiology and No. 20029 from the Japanese Society for Disability and Oral Health). This study was conducted in accordance with the Declaration of Helsinki. Participation in the study was voluntary and anonymous. Participating members gave their informed consent by voluntarily responding to the survey.

\section{Consent for publication}

Not applicable.

\section{Competing interests}

The authors declare that they have no competing interests.

\section{Author details}

${ }^{1}$ Section of Anesthesiology, Department of Diagnostics and General Care, Fukuoka Dental University, Fukuoka, Japan. ${ }^{2}$ Department of Special Care
Dentistry, Nagasaki University Hospital, Nagasaki, Japan. ${ }^{3}$ Department of Dental Anesthesiology, Hokkaido University, Hokkaido, Japan. ${ }^{4}$ Clinical Research Center, Nagasaki University Hospital, Nagasaki, Japan. ${ }^{5}$ Department of Dental Anesthesiology, Nagasaki University Institute of Biomedical Sciences, Course of Medical and Dental Sciences, Nagasaki, Japan.

Received: 17 September 2021 Accepted: 3 February 2022 Published online: 11 February 2022

\section{References}

1. McNeil D, Randall C. Dental fear and anxiety associated with oral health care: conceptual and clinical issues. In: Mostofsky DI, editor. Fortune F Behavioral dentistry. 2nd ed. Ames: Blackwell; 2014. p. 89-107.

2. Appukuttan DP. Strategies to manage patients with dental anxiety and dental phobia: literature review. Clin Cosmet Investig Dent. 2016:8:35-50.

3. Armfield JM, Heaton LJ. Management of fear and anxiety in the dental clinic: a review. Aust Dent J. 2013;58:390-7.

4. Fredrikson M, Annas P, Fischer H, Wik G. Gender and age differences in the prevalence of specific fears and phobias. Behav Res Ther. 1996;34:33-9.

5. Stinson FS, Dawson DA, Patricia Chou S, Smith S, Goldstein RB, June Ruan $W$, et al. The epidemiology of DSM-IV specific phobia in the USA: results from the National Epidemiologic Survey on Alcohol and Related Conditions. Psychol Med. 2007;37:1047-59.

6. Oosterink FM, de Jongh A, Hoogstraten J. Prevalence of dental fear and phobia relative to other fear and phobia subtypes. Eur J Oral Sci. 2009;117:135-43.

7. Dailey YM, Humphris GM, Lennon MA. Reducing patients'state anxiety in general dental practice: a randomized controlled trial. J Dent Res. 2002:81:319-22.

8. Hally J, Freeman R, Yuan S, Humphris G. The importance of acknowledgement of emotions in routine patient psychological assessment: the example of the dental setting. Patient Educ Couns. 2017;100:2102-5.

9. Humphris GM, Morrison T, Lindsay SJ. The Modified Dental Anxiety Scale: validation and United Kingdom norms. Community Dent Health. 1995; 12:143-50.

10. King $\mathrm{KH}$, Humphris $\mathrm{GM}$. Evidence to confirm the cut-off for screening dental phobia using the Modified Dental Anxiety Scale. Soc Sci Dent. 2010;1:21-8

11. Ogawa M, Sago T, Furukawa $H$. The reliability and validity of the Japanese version of the modified dental anxiety Scale among dental outpatients. Sci World J. 2020;2020:8734946.

12. Yuan S, Freeman R, Lahti S, Lloyd-Williams F, Humphris G. Some psychometric properties of the Chinese version of the Modified Dental Anxiety Scale with cross validation. Health Qual Life Outcomes. 2008;6:22.

13. Coolidge T, Hillstead MB, Farjo N, Weinstein P, Coldwell SE. Additional psychometric data for the Spanish Modified Dental Anxiety Scale, and psychometric data for a Spanish version of the Revised Dental Beliefs Survey. BMC Oral Health. 2010;10:12.

14. Kankaala T, Laine $H$, Laitala ML, Rajavaara $P$, Vähänikkilä $H$, Pesonen $P$, et al. 10-year follow-up study on attendance pattern after dental treatment in primary oral health care clinic for fearful patients. BMC Oral Health. 2021;21:522.

15. Wide $U$, Hakeberg M. Treatment of dental anxiety and phobia-diagnostic criteria and conceptual model of behavioural treatment. Dent J (Basel). 2021;9:153.

16. Wanyonyi KL, White S, Gallagher JE. Conscious sedation: is this provision equitable? Analysis of sedation services provided within primary dental care in England, 2012-2014. BDJ Open. 2016;2:16002.

17. Kani E, Asimakopoulou K, Daly B, Hare J, Lewis J, Scambler S, et al. Characteristics of patients attending for cognitive behavioural therapy at one UK specialist unit for dental phobia and outcomes of treatment. Br Dent J. 2015;219:501-6.

18. Davies JG, Wilson KI, Clements AL. A joint approach to treating dental phobia: a re-evaluation of a collaboration between community dental services and specialist psychotherapy services ten years on. Br Dent J. 2011;211:159-62.

19. Amano I, Maehama W, Toshimitsu T, Tasaki S, Ozaki A, Kaji C, et al. A study on the application of intravenous sedation and General Anesthesia to patients with special needs. J Jpn Soc. 2017;38:85-90. 
20. Harano N, Sago T, Nunomaki M, Nakatsu Y, Yamaguchi K, Shiiba S, et al. A study of behavior management for a patient with difficulty in cooperating with dental treatment in our clinic. J Jpn Soc. 2017;38:64-8.

21. Dailey YM, Humphris GM, Lennon MA. The use of dental anxiety questionnaires: a survey of a group of UK dental practitioners. Br Dent J. 2001;190:450-3.

22. Armfield JM, Mohan H, Luzzi L, Chrisopoulos S. Dental anxiety screening practices and self-reported training needs among Australian dentists. Aust Dent J. 2014:59:464-72.

23. Model Core Curriculum for Dental Education. Revision. Model core curriculum revision coordination committee. p. AY2016; 2018. https://www. mext.go.jp/component/a_menu/education/detail/_icsFiles/afieldfile/ 2018/06/18/1325989_31.pdf. Accessed 2 Dec 2021.

24. Fallea A, Zuccarello R, Cali F. Dental anxiety in patients with borderline intellectual functioning and patients with intellectual disabilities. BMC Oral Health. 2016;16:114.

\section{Publisher's Note}

Springer Nature remains neutral with regard to jurisdictional claims in published maps and institutional affiliations.

- fast, convenient online submission

- thorough peer review by experienced researchers in your field

- rapid publication on acceptance

- support for research data, including large and complex data types

- gold Open Access which fosters wider collaboration and increased citations

- maximum visibility for your research: over 100M website views per year

At BMC, research is always in progress.

Learn more biomedcentral.com/submissions 